(c) American Dairy Science Association, 2004.

\title{
Hot Topic: Using a Stearoyl-CoA Desaturase Transgene to Alter Milk Fatty Acid Composition
}

\author{
W. A. Reh, ${ }^{1}$ E. A. Maga, ${ }^{1}$ N. M. B. Collette, ${ }^{1}$ A. Moyer, ${ }^{1}$ J. S. Conrad-Brink, ${ }^{1}$ \\ S. J. Taylor, ${ }^{1}$ E. J. DePeters, ${ }^{1}$ S. Oppenheim, ${ }^{1}$ J. D. Rowe, ${ }^{2}$ R. H. BonDurant, ${ }^{2}$ \\ G. B. Anderson, ${ }^{1}$ and J. D. Murray ${ }^{1,2}$ \\ ${ }^{1}$ Department of Animal Science and \\ 2Department of Population Health and Reproduction, \\ University of California, Davis 95616
}

\begin{abstract}
Stearoyl-CoA desaturase enzyme converts specific medium- and long-chain saturated fatty acids to their monounsaturated form. Transgenic goats expressing a bovine $\beta$-lactoglobulin promoter-rat stearoyl-CoA desaturase cDNA construct in mammary gland epithelial cells were produced by pronuclear microinjection. The fatty acid composition of milk from 4 female transgenic founders was analyzed on $\mathrm{d} 7,14$, and 30 of their first lactation. In 2 animals, the expression of the transgene changed the overall fatty acid composition of the resulting milk fat to a less saturated and more monounsaturated fatty acid profile at d 7 of lactation; however, this effect diminished by d 30. In addition, one animal had an increased proportion of the rumen-derived monounsaturated fatty acid C18:1 trans 11 converted by stearoyl-CoA desaturase to the conjugated linoleic acid isomer $\mathrm{C} 18: 2$ cis 9 trans 11 . Milk that has higher proportions of monounsaturated fatty acids and conjugated linoleic acid may have benefits for human cardiovascular health.
\end{abstract}

(Key words: stearoyl-CoA desaturase, transgenic, milk fat, conjugated linoleic acid)

Abbreviation key: $\mathbf{B l g}=\beta$-lactoglobulin, $\mathbf{C L A}=$ conjugated linoleic acid, FA = fatty acid, MUFA = monounsaturated fatty acid, PUFA = polyunsaturated fatty acid, $\mathbf{S C D}=$ stearoyl-CoA desaturase, $\mathbf{S F A}=$ saturated fatty acid.

Results of many epidemiologic, clinical, and animal studies indicate that high quantities of dietary fat, especially saturated fatty acids (SFA), can lead to an increase of blood cholesterol and consequently increase risk for atherosclerosis and coronary heart disease in

\footnotetext{
Received February 24, 2004.

Accepted May 20, 2004.

Corresponding author: J. D Murray; e-mail: jdmurray@ucdavis.
} edu. humans (German et al., 1997; Kromhout et al., 2002). A diet that minimizes cardiovascular risk factors and contributes to an overall reduction of diseases not cardiovascular in nature (such as diabetes) could be achieved by changing the overall composition of dietary fat (Nestel, 1995). Both mono- (MUFA) and polyunsaturated (PUFA) fatty acids affect cardiac risk by lowering serum cholesterol, while SFA raise both serum cholesterol and low density lipoprotein cholesterol, factors most associated with risk of coronary heart disease (Nestel, 1995; Menotti, 1999). As approximately onethird of the SFA in American diets come from the consumption of dairy products (Havel, 1997), changing the fatty acid (FA) composition of milk is a worthwhile objective.

In 1991, Grummer suggested an "ideal" nutritional milk fat based on dietary recommendations would consist of approximately 10\% PUFA, 8\% SFA, and $82 \%$ MUFA. This greatly differs from the typical cows' milk FA composition of $5 \%$ PUFA, 70\% SFA, and 25\% MUFA. Such a compositional change as this cannot be accomplished by solely altering the diet of lactating dairy cows or goats, as consumed unsaturated FA are extensively biohydrogenated in the rumen prior to absorption in the small intestine (Doreau et al., 1997). The hydrogenation of unsaturated FA by rumen bacteria results in milk FA being more saturated than usual dietary FA intake.

In ruminants, the effect of intestinal absorption of high amounts of long-chain SFA on milk FA composition is partially offset by the activity of the stearoylCoA desaturase (SCD) enzyme in mammary gland epithelial cells. Stearoyl-CoA desaturase is responsible for the oxidation reaction converting SFA to MUFA by the addition of a cis double bond between carbons 9 and 10 of some medium- and long-chain FA, primarily palmitate $(16: 0)$ to palmitoleate $(16: 1)$ and stearate $(\mathrm{C} 18: 0)$ to oleate (C18:1) (Tocher et al., 1998). If SCD activity was increased in the mammary gland, the total amount of MUFA could be increased, thus resulting in a decrease in the amount of SFA in milk. 
In addition to overall quantity of SFA, a group of specific FA known as conjugated linoleic acids (CLA) that occur in ruminant milk are also of interest in terms of human health. Conjugated linoleic acid, principally the 18:2 cis 9 trans 11 isomer, may be anticarcinogenic and anti-atherosclerotic, decrease fat accumulation, and can modulate the immune response and thus reduce the cachetic response, enhance cell-mediated responses, and decrease the inflammatory response (Pariza et al., 1999; MacDonald, 2000). Furthermore, CLA can be produced by the action of SCD on the 18:1 trans 11 product of rumen bacteria by the addition of a double bond in the cis position at C9, and this activity has been established in the mammary gland (Griinari et al., 2000; Santora et al., 2000).

The ability to genetically engineer the mammary gland to alter properties of milk, coupled with the difficulties of altering milk FA composition by simple dietary manipulation, suggests transgenesis as an alternate approach for changing overall milk FA composition. An SCD transgene would present a permanent and heritable means to improve the nutritional and healthful attributes of this important food. Furthermore, milk from transgenic animals expressing SCD transgenes should pose minimal health risks when consumed by humans as SCD is already expressed in mammary gland cells, it is a membrane-bound enzyme, and no new FA are being introduced into the milk; nor are any being completely removed.

To test this hypothesis, we generated transgenic dairy goats as a model for the dairy cow with a DNA construct designed to express the rat SCD cDNA in the mammary gland under control of the bovine $\beta$-lactoglobulin (Blg) promoter. The 5526-bp bovine Blg-rat SCD construct (pBlgSCD) consisted of $1216 \mathrm{bp}$ of the bovine Blg promoter (from the EagI site at -954 Accession \#X14710) through untranslated exon 1 and the start codon. The start codon was replaced by a XhoI site and was followed by $1831 \mathrm{bp}$ of bovine Blg DNA from +4 to the ClaI site at 4044 and $1349 \mathrm{bp}$ from the untranslated portion of exon 6 through the $S p h$ I site at 7639 , including $917 \mathrm{bp}$ of $3^{\prime}$ flanking DNA. The 1079-bp rat SCD cDNA open reading frame containing its own start and stop codons was inserted at the $X h o I$ site to create pBlgSCD. The construct was excised from the plasmid using EagI and SphI, purified using Elutip columns (Scheicher \& Schuell), and diluted to $5 \mathrm{ng} / \mu \mathrm{L}$ in microinjection buffer (10 $\mathrm{m} M$ Tris, $0.25 \mathrm{~m} M$ EDTA, $\mathrm{pH}$ 7.4).

Goats were chosen for this study as they are ruminant animals and respond to diet in a fashion similar to a cow. Furthermore, goats have a milk fat profile that closely resembles that of the cow, making them a relevant bovine model. Transgenic goats were produced with DNA construct $\mathrm{pBlgSCD}$ by standard pronuclear microinjection procedures with and without RecA coating as previously described (Maga et al., 2003). Pregnancies were carried to term, and all offspring born were screened for the presence of the transgene with construct-specific PCR primers BLA-5'F (5'CCTGTCCTTGTCTAAGAGG-3') and RSCD3 (5'-AATATCCCCCAGAGCAAGGT-3') that generate a 634-bp diagnostic fragment. The PCR-positive founders were confirmed by Southern analysis. The goats used were outbred dairy goats, principally of the Toggenburg breed. Thirteen transgenic founders were identified (7 does and 6 bucks) out of 37 kids born and analyzed. To date, all 7 transgenic founders (6 female, 1 male) that have been bred have transmitted the transgene to some of their offspring (data not shown).

Reverse transcriptase-PCR was used to detect the presence of transgene-specific mRNA in total RNA isolated from mammary gland tissue taken from 5 female founders $(31,34,37,51$, and 53$)$ by biopsy during wk 30 of lactation. Total RNA was extracted from mammary tissue using Trizol reagent (Invitrogen). For reverse transcriptase-PCR, $1 \mu \mathrm{g}$ of total RNA was first DNase treated and then used to generate cDNA by reverse transcription using oligonucleotide (dT)18 as primer. Resulting cDNA was diluted 1:10 and utilized for PCR. Two control reactions were run for each $\mathrm{RT}$ reaction to ensure that reagents were not contaminated and that positive RT-PCR results were in fact $\mathrm{cDNA}$ and not contaminating genomic DNA. The first control was an RT reaction with no RNA to test the purity of reagents. The second control was an RT reaction with diethylpyrocarbonate water rather than reverse transcriptase, to verify complete DNase treatment. The PCR amplification of the cDNA was performed using primers specific to the transgene cDNA and spanned the $5^{\prime}$ junction of bBLG exon 1 and rSCD and yielded a 792-bp product specific to the transgene message (forward primer BLG-E1 5'-CAGAGCTCAGAAGCGTGACC-3'; reverse primer -RSCD-4R 5'-GATGAAGCACATGAGCAGGA$\left.3^{\prime}\right)$. The BlgSCD mRNA was detected in goats 34,37 , 51 , and 53, while goat 31 appeared to have ceased expression of the transgene at the time of this analysis (Figure 1). The BlgSCD mRNA appeared to be unstable, or present in very low amounts, as expression could not be detected by Northern blotting. Of these expressing animals, 2 (51 and 53) were generated by the injection of RecA-coated DNA.

Four female transgenic founders and 10 nontransgenic breed and parity matched control animals were bred to nontransgenic males, and milk was collected on d 7, 14, and 30 of the first lactation for FA composition analysis of milk fat. Goats 24 and 31 were not lactating at the time of milk collection and were not included in this analysis. Milk from each goat was 


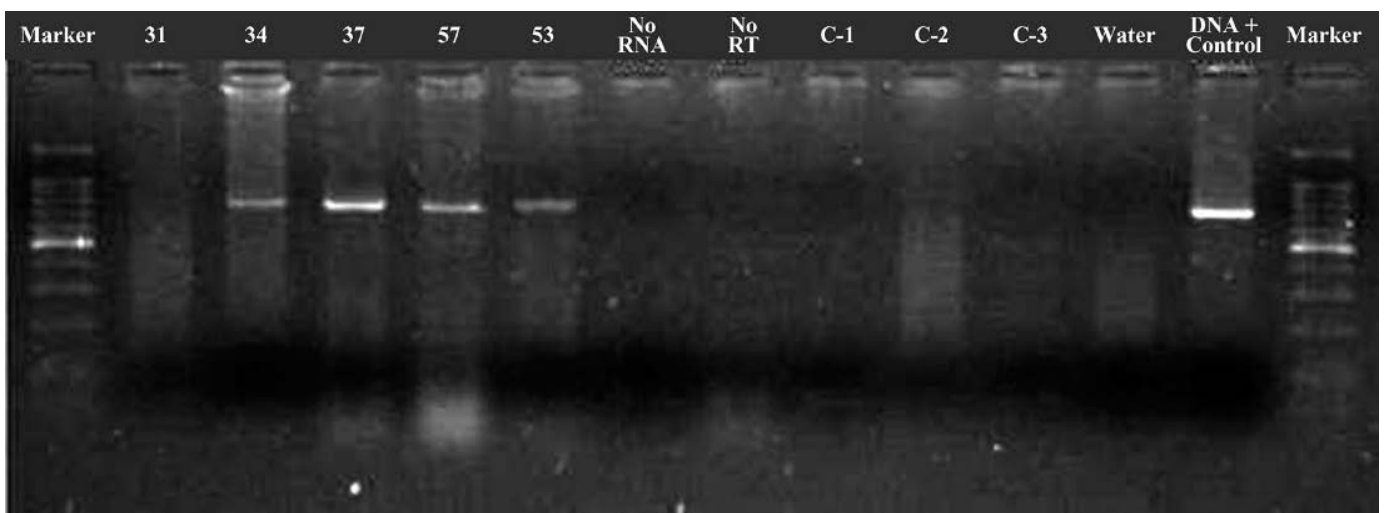

Figure 1. Reverse transcription-PCR of desaturase expression in mammary gland biopsies. Expressing animals should contain a 792 bp band. Lanes 1 to 5 contain RT-PCR product from lactating transgenic animals 31,34, 37, 51, and 53, respectively. Lanes 6 and 7 are RT-PCR control of no RNA and no RT, respectively. Lanes 8 to 10 contain RT-PCR product from lactating, nontransgenic controls (C-1, C2, and C-3), and lane 11 and 12 are PCR controls of no sample (water) and a DNA positive control, respectively.

collected from the evening milking and again the following morning. Total milk yield was quantified by weight, with evening and morning samples mixed proportionately to represent one full day's milk production. Milk fat and milk protein contents were determined by the California Dairy Herd Improvement Association laboratory using the standard DHIA analysis (Bentley). The percentage of protein and fat in milk from the 4 transgenic founders fell in the same range as the means of our dairy goat herd for both components, indicating that expression of the transgene did not change the gross composition of the milk (data not shown).

The FA composition of whole transgenic and nontransgenic milk was determined by gas chromatography as previously described (DePeters et al., 2001). Briefly, extracted milk fat was methylated using $3 \mathrm{M}$ potassium hydroxide and quantified using external standards acquired from Nu-Chek-Prep (Elysian, MN), containing a known amount of each FA of interest. The methyl esters were then resolved using a Hewlett Packard 5890 gas chromatograph equipped with a Supelco (Bellefonte, PA) 2560 capillary column (100 m long, 0.25 $\mathrm{mm}$ i.d., 0.20 micron film thickness).

In addition to determining the weight percentage of individual FA, product-to-substrate ratios were used to estimate activity of the SCD enzyme. Each primary transgenic goat analyzed represents a unique transgenic line, precluding the combining of the results from different transgenic animals. For these reasons, the data from the transgenic animals were compared to the mean plus and minus 2 and 3 standard deviations for the same FA or product:precursor ratios obtained for the 10 nontransgenic controls. Values from transgenic animals falling 2 or more SD from the mean of the control groups were accepted as signifying possible significant alterations in FA composition. Individual weight percentage values for selected $\mathrm{FA}, \mathrm{FA}$ ratios (14:1/14:0, 16:1/16:0, 18:1/18:0, 18:2 cis 9 trans11/18:1 trans 11), and overall FA profiles for 4 of the transgenic founders and 10 control animals sampled are given in Table 1 for $\mathrm{d} 7$ and 30 of lactation.

The milk FA composition was not consistent for each of the goats. This should be expected because each animal is a unique transgenic line and will differ in transgene copy number, integration sites, overall expression, and thus phenotypic effects. However, it is clear from the results that the transgene had a dramatic influence on milk FA profiles in 2 of the 4 transgenic animals (34 and 37) at $\mathrm{d} 7$ of lactation (Table1). They had expected increases in 18:1 and 16:1 with all percentages over or approaching $3 \mathrm{SD}$ from the control mean. These animals also had significant changes in overall FA profiles with SFA percentages 3 SD lower and MUFA percentages 3 SD higher than control means. Medium-chain FA (10:0, 12:0, and 14:0) were also decreased in these goats. By d 14 and 30 of lactation it was apparent that the transgene's effects were diminishing. On d 14 (data not shown), goats 34 and 37 had similar profiles as d 7, although results were not significant, with the exception of 37 having 2 SD higher MUFA and lower 10:0 and 12:0. At d 30, these goats still had FA percentages at noteworthy levels (Table 1) as well as increased FA ratios, indicating that the SCD transgene may have still been having an effect, though clearly not to the extent of $d$. Conversely, goats 51 and 53 had FA profiles similar to those of the controls at all 3 time points, although 51 did have significant increases in amounts of 18:2 cis 9 trans 11 CLA (Table 1 ). The difference in the transgene's effect may be related to differing expression levels in the various founders. 
Table 1. Weight percentage (g FA/100 g fat) of selected FA in the milk from d 7 and 30 of lactation in 4 SCD transgenic does and 10 breed, age, parity, and d of lactation matched controls.

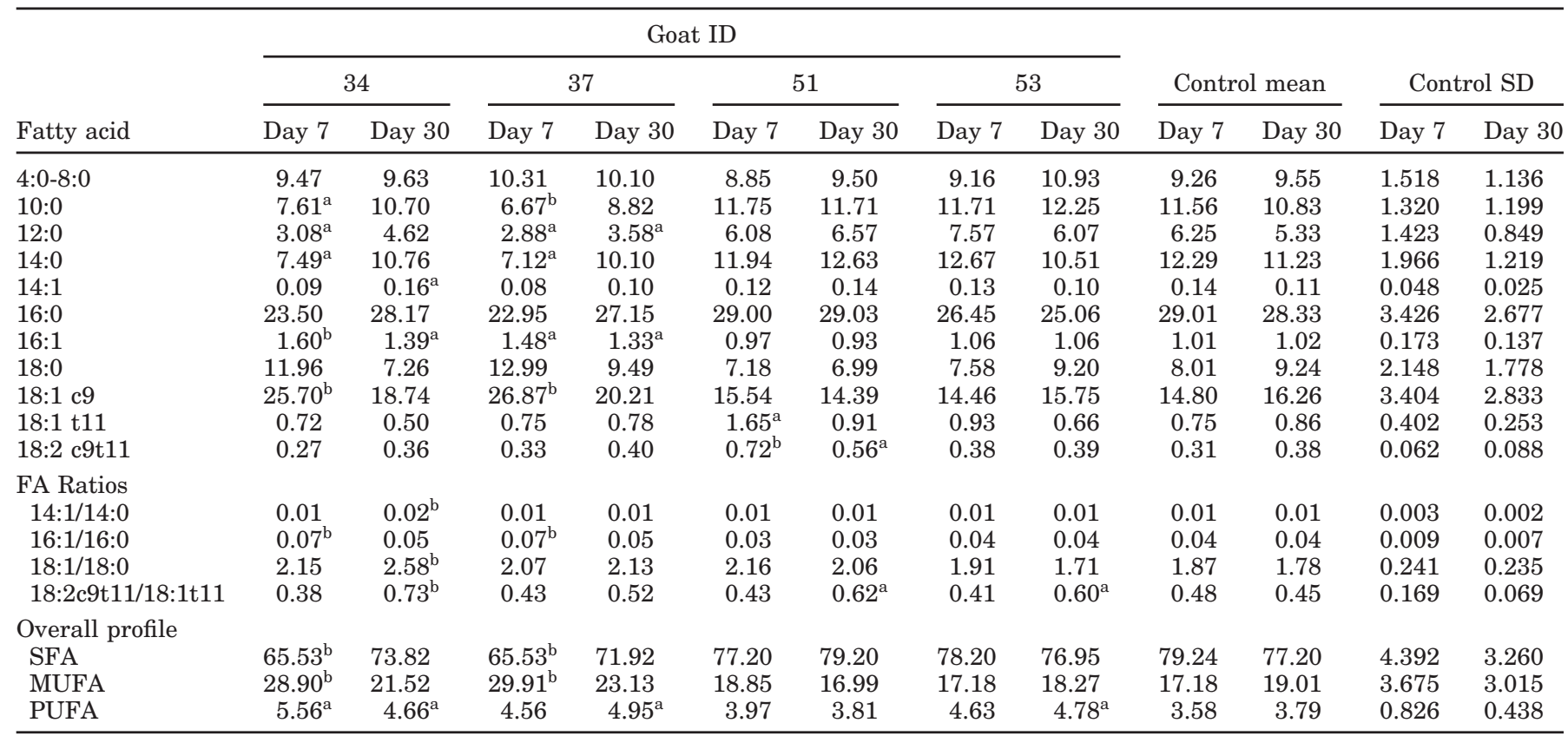

${ }^{a}$ Values are either greater than or less than the control mean plus or minus 2 standard deviations.

${ }^{b}$ Values are either greater than or less than the control mean plus or minus 3 standard deviations.

We believe that some of the dramatic differences observed in the FA profile of 34 and 37 at $d 7$ may be due to a secondary effect of the transgene that decreased de novo FA synthesis in the mammary gland resulting in increased MUFA and PUFA concentrations. The increase of d 30 PUFA in 34, 37, and 53 may also be explained by such activities. Evidence of this is the significant decrease in the percentage of mammary gland derived medium-chain FA (10:0, 12:0, and 14:0) and increase (data not shown) in blood derived FA (17:0, linoleic acid, linolenic acid, and $>20$ carbon FA). This effect may be due to the competition of the SCD for energy sources, physical interference with the malonylCoA FA elongation pathway, or other interactions between pathways.

The transient nature of our transgene's effects is probably due to unstable expression of our transgene. Results from previous studies using this same transgene construct in mice (Reh, 2002) suggest that the BlgSCD-derived message is highly unstable and degraded very rapidly. These studies also revealed that this transgene ceased expressing in these mice. Expression in our goats also supports this as mRNA levels of the transgene by wk 30 of lactation were extremely low. The potential exists to increase the magnitude of the FA composition changes seen in these transgenic animals. Improvements in transgene expression levels could be made by using a stronger promoter such as the $\alpha_{\mathrm{s} 1^{-}}$ casein promoter that previously supported high, stable levels of expression (Maga et al., 1994). Additionally, work is in progress to determine whether there are mRNA stabilization elements, which were absent from our original transgene, present in the $3^{\prime}$ untranslated region of the rSCD mRNA that can contribute to increased message stability when incorporated into future transgene constructs.

Extensive medical and nutritional research indicate that there are potential benefits of reducing SFA and increasing MUFA and CLA intake in human diets. The current work provides preliminary evidence for proof of principle that a SCD-based mammary gland-specific transgene can be utilized to alter the FA composition of milk fat from dairy animals. The methods of genetic engineering, alternative animal diets, and selective animal breeding can be used individually or in combination to further improve the healthful benefits of consuming dairy products.

\section{ACKNOWLEDGMENTS}

We thank the UC Davis Dairy Goat Facility managers during the period of this work, La Donna Foley and Jan Carlson for their excellent assistance in obtaining goats, programming reproduction, and care of the animals. Special thanks go to Mandy Hamilton, Erika Scharfen, Jolene Berg, and Harmony McPhearson for 
the hard work and long nights they put in on our behalf. We also express our gratitude to the many other undergraduate and D.V.M. students who assisted with the production of the transgenic goats and their subsequent care. We thank Denae Wagner, Mike Lane, and other members of the School of Veterinary Medicine team that assisted with embryo transfers, monitoring pregnancies, and general veterinary care of our animals. Milk fatty acid analyses were performed by the Dairy Milk Components Laboratory with funding from the California Dairy Research Foundation, Davis.

\section{REFERENCES}

DePeters, E. J., J. B. German, S. J. Taylor, S. T. Essex, and H. PerezMonti. 2001. Fatty acid and triglyceride composition of milk fat from lactating Holstein cows in response to supplemental canola oil. J. Dairy Sci. 84:929-936.

Doreau, M., D. I. Demeyer and C. J. Van Nevel. 1997. Transformations and effects of unsaturated fatty acids in the rumen. Consequences on milk fat secretion. Pages 73-92 in Milk Composition, Production, and Biotechnology. R. A. S. Welch, D. J. W. Burns, S. R. Davis, A. I. Popay, and C. J. Prosser, ed. CAB International, Wallingford, UK.

German, J. B., L. Morand, C. J. Dillard, and R. Xu. 1997. Milk fat composition: Targets for alteration of function and nutrition. Pages 35-72 in Milk Composition, Production, and Biotechnology. R. A. S. Welch, D. J. W. Burns, S. R. Davis, A. I. Popay and C. J. Prosser, eds. CAB International, Wallingford, UK.

Griinari, J. M., B. A. Corl, S. H. Lacy, P. Y. Chouinard, K. V. Nurmela, and D. E. Bauman. 2000. Conjugated linoleic acid is synthesized endogenously in lactating dairy cows by $\Delta 9$-desaturase. J. Nutr. 130:2285-2291.
Grummer, R. R. 1991. Effect of feed on the composition of milk fat. J. Dairy Sci. 74:3244-3257.

Havel, R. J. 1997. Milk fat consumption and human health: Recent NIH and other American government recommendations. Pages 13-22 in Milk Composition, Production, and Biotechnology. R. A. S. Welch, D. J. W. Burns, S. R. Davis, A. I. Popay and C. J. Prosser, ed. CAB International, Wallingford, UK.

Kromhout, D., A. Menotti, H. Kestleloot, and S. Sans. 2002. Prevention of coronary heart disease by diet and lifestyle: Evidence from prospective cross-cultural, cohort, and interventional studies. Circulation 105:893-898.

MacDonald, H. B. 2000. Conjugated linoleic acid and disease prevention: A review of current knowledge. J. Am. Coll. Nutr. 19:111S-118S.

Maga, E.A., G. B. Anderson, and J. D. Murray. 1994. Expression of human lysozyme mRNA in the mammary gland of transgenic mice. Transgenic Res. 3:36-42.

Maga, E. A., R. G. Sargent, H. Zeng, S. Pati, D. A. Zarling, S. M. Oppenheim, N. M. Collette, A. L. Moyer, J. S. Conrad-Brink, J. D. Rowe, R. H. BonDurant, G. B. Anderson, and J. D. Murray. 2003. Increased efficiency of transgenic livestock production. Transgenic Res. 12:485-496.

Menotti, A. 1999. Diet cholesterol and coronary heart disease: A perspective. Acta Cardiol. 54:169-172.

Nestel, P. J. 1995. Controlling coronary risk through nutrition. Can. J. Cardiol. 11:9G-14G.

Pariza, M. W., Y. Park, and M. E. Cook. 1999. Conjugated linoleic acid and the control of cancer and obesity. Toxicolog. Sci. 52 (Suppl.):107-110.

Reh, W. A. 2002. Alteration of milk fat composition and transgene silencing in mice carrying a bovine $\beta$-lactoglobulin-rat stearoylCoA desaturase transgene. M. S. Thesis, Univ. of California, Davis.

Santora, J. E., D. L. Palmquist, and K. L. Roehrig. 2000. Transvaccenic acid is desaturated to conjugated linoleic acid in mice. J. Nutr. 130:208-215.

Tocher, D. R., M. J. Leaver, and P. A. Hodgson. 1998. Recent developments in the molecular biology and biochemistry of fatty acyl desaturases. Prog. Lipid Res. 37:73-117. 